\title{
Variational analysis of the Rashba splitting in III-V semiconductor inversion layers
}

\author{
M. A. Toloza Sandoval and A. Ferreira da Silva \\ Instituto de Física, Universidade Federal da Bahia, 40210-340 Salvador, Bahia, Brazil
}

E. A. de Andrada e Silva*

Instituto Nacional de Pesquisas Espaciais, INPE, C.P. 515, 12201-970 São José dos Campos, São Paulo, Brazil

G. C. La Rocca

Scuola Normale Superiore and CNISM, Piazza dei Cavalieri 7, I-56126 Pisa, Italy

(Received 7 May 2010; revised manuscript received 6 March 2011; published 10 June 2011)

\begin{abstract}
A spin-dependent variational theory is used to analyze the Rashba spin-orbit splitting in two-dimensional electron gases formed in III-V semiconductor inversion layers. The spin split conduction subbands in $\mathrm{CdTe} / \mathrm{InSb}$, insulator/InAs, InP/InGaAs, InAlAs/InGaAs, and $\mathrm{AlGaAs} / \mathrm{GaAs}$ heterojunctions are calculated. The theory, presented here in detail, is based on the $8 \times 8 \mathbf{k} \cdot \mathbf{p}$ Kane model and on the introduction of simple and convenient spin-dependent Fang-Howard trial functions, and leads to analytical expressions for the split subbands, as well as allows for a detailed knowledge of the Rashba spin-orbit coupling, including its explicit dependence on structure parameters and its decomposition into separate contributions. The Rashba coupling parameter and the population difference in the spin-split subbands, as experimentally determined from the beating pattern of the Shubnikov-de Haas $(\mathrm{SdH})$ oscillations, are obtained as a function of the electron density $\left(n_{s}\right)$. The separate contributions to the particularly large Rashba splitting in CdTe/InSb heterojunctions are also computed and discussed. It is shown, for example, that due to the spin-dependent boundary conditions, the direct Rashba spin-orbit coupling term in the effective Hamiltonian dominates the splitting only for $n_{s}>10^{10} \mathrm{~cm}^{-2}$ while it is the barrier penetration kinetic energy term that gives the largest contribution to the Rashba effect at lower densities.
\end{abstract}

DOI: $10.1103 /$ PhysRevB.83.235315

PACS number(s): 71.70.Ej, 71.15.Ap, 73.21.Fg, 85.75.-d

\section{INTRODUCTION}

A main trend in semiconductor spintronics concerns the manipulation of the electron spin with the use of electric fields only, i.e., without external magnetic fields or ferromagnetic materials. ${ }^{1}$ This is possible with the use of the spin-orbit coupling. In particular, the Rashba effect in III-V semiconductor inversion layers has been studied much, since it allows for a direct and convenient gate-voltage control of the electron spin precession in the two-dimensional electron gases (2DEGs) conducting channels. The effect can be described by means of an effective magnetic field seen by the moving electron due to the Lorentz transformed electric field. However, the calculation or measurement of such magnetic field at semiconductor heterointerfaces is a complex and unsolved problem. In particular, the effects of band offset and barrier penetration, connected to the boundary conditions at the interface, have been somewhat controversial. ${ }^{2,3}$ In order to better understand and apply the effect, further analysis is necessary. In particular, the decomposition of the resulting spin splitting into separate contributions would be very helpful. However, this is not a simple task with most of the model calculations available to study the Rashba effect. ${ }^{4}$

A spin-dependent variational solution within standard envelope function approximation was recently developed, which is in good agreement with both the experimental results and the exact numerical calculations, and able to analytically distinguish the different contributions to the Rashba coupling in III-V semiconductor inversion layers. ${ }^{5}$ Here we apply this theory to analyze the Rashba effect in III-V semiconductor inversion layers. We calculate the
Rashba coupling parameter, study its composition, and present specific results for $\mathrm{CdTe} / \mathrm{InSb}$, insulator/InAs, InP/InGaAs, InAlAs/InGaAs, and AlGaAs/GaAs systems, as a function of the 2DEG electron density. The corresponding population difference in the split subbands, as experimentally determined from the beating pattern of the $\mathrm{SdH}$ oscillations, is also obtained. The theory is shown to well reproduce the main known properties of the Rashba effect and thus to improve our knowledge about it. As an example, the particularly large Rashba splitting in $\mathrm{CdTe} / \mathrm{InSb}$ heterojunctions is shown to be dominated by the usual (direct) Rashba spin-orbit term in the effective Hamiltonian only for electron densities higher than $10^{10} \mathrm{e} / \mathrm{cm}^{2}$. For lower densities, the barrier penetration kinetic energy term becomes the dominant one. The theory is presented here with important details which were missing in Ref. 5, like intermediate analytical results for the energy integrals and directions for the correct use of its parameters.

In 2DEGs formed at such junctions, the conducting electrons are confined near the interface by an electric field $\mathcal{E}$ plus the barrier due to the conduction band offset $v_{0}$, which form an approximate triangular potential. The confining electric field, and therefore the resulting Rashba coupling parameter, can be modulated by the gate voltage or carrier density $\left(n_{s}\right)$ of the 2DEG. The physics of such system has been much studied, mostly with the effective 2D Hamiltonian $H_{c}=\hbar^{2} k_{\|}^{2} / 2 m^{*}+\alpha^{*} \boldsymbol{\sigma} \cdot \mathbf{k} \times \mathbf{e}_{z}$, with $\mathbf{k}_{\|}=k_{x} \mathbf{e}_{x}+k_{y} \mathbf{e}_{y}$. The resulting Rashba splitting at the Fermi energy is then simply given by $\delta \varepsilon=2 \alpha^{*} k_{F}$. In the infinite barrier approximation, simple expressions for the Rashba coupling parameter $\left(\alpha^{*}\right)$ can be derived from $8 \times 8 \mathbf{k} \cdot \mathbf{p}$ Kane model, ${ }^{6}$ dependent only on the band parameters of the well material. The present work 
extends this scheme in order to add the contributions due to the barrier penetration and to the spin-dependent boundary conditions. We first review the variational theory in Ref. 5, starting with the $8 \times 8$ Kane model applied to heterojunctions, and then discussing the perturbation expansions used to derive renormalized band parameters of the effective Hamiltonian, and its variational solution with the introduction of spinresolved Fang-Howard trial functions. Finally, we present and discuss the results for the Rashba coupling parameter and population difference in the split subbands of different III-V semiconductor heterojunctions, as a function of the total $2 \mathrm{DEG}$ carrier density.

\section{KANE MODEL FOR HETEROJUNCTIONS}

It is well known that, with an appropriate choice of spin quantization direction (i.e., $\mathbf{k}_{\|} \times \mathbf{e}_{z}$ ), the $8 \times 8$ effective Hamiltonian based on Kanes's model can be block diagonalized into two $4 \times 4$ blocks, one for each of the two Rashba split electron subbands. ${ }^{6-8}$ The equations corresponding to each block can be resolved (downfolded) for the conduction band envelope function $\Psi$, which is then seen to be the eigenfunction of the following effective Hamiltonian, in which the Rashba coupling (the spin-dependent term below, also called direct Rashba coupling term) is in a simple form:

$$
\begin{aligned}
H_{\mathrm{eff} \pm}= & -\frac{\hbar^{2}}{2} \frac{d}{d z} \frac{1}{m\left(z, \varepsilon_{ \pm}\right)} \frac{d}{d z}+\frac{\hbar^{2} k_{\|}^{2}}{2 m\left(z, \varepsilon_{ \pm}\right)}+E_{c}(z) \\
& +U(z) \mp\left[\frac{d}{d z} \beta\left(z, \varepsilon_{ \pm}\right)\right] k_{\|}
\end{aligned}
$$

with

$$
\begin{aligned}
\frac{1}{m\left(z, \varepsilon_{ \pm}\right)}= & \frac{P^{2}}{\hbar^{2}}\left[\frac{2}{\varepsilon_{ \pm}-U(z)-E_{v}(z)}\right. \\
& \left.+\frac{1}{\varepsilon_{ \pm}-U(z)-E_{v}(z)+\Delta(z)}\right]
\end{aligned}
$$

and

$$
\begin{aligned}
\beta\left(z, \varepsilon_{ \pm}\right)= & \frac{P^{2}}{2}\left[\frac{1}{\varepsilon_{ \pm}-U(z)-E_{v}(z)}\right. \\
& \left.-\frac{1}{\varepsilon_{ \pm}-U(z)-E_{v}(z)+\Delta(z)}\right],
\end{aligned}
$$

where $z$ is the growth direction, $E_{c}, E_{v}$, and $\Delta$, respectively, stand for the conduction band edge, the valence band edge, and the valence band spin-orbit splitting, and $U(z)$ is the electrostatic potential energy. The \pm signs correspond to spin up and down along the spin quantization direction; $\varepsilon_{ \pm}=\varepsilon_{ \pm}\left(k_{\|}\right)$give the spin-split subband dispersion relations we are looking for; and $P$ is the usual interband momentum matrix element, which is assumed to be constant and is calculated with the measured conduction band effective mass in the well material. The corresponding boundary conditions at the interface are that the envelope functions $\Psi_{ \pm}$must be continuous, as well as

$$
-\frac{\hbar^{2}}{2 m} \frac{d}{d z} \Psi_{ \pm} \mp \beta k_{\|} \Psi_{ \pm} .
$$

These are the so-called spin-dependent boundary conditions for the spin-dependent envelope functions.

Note that in this model, the $\mathbf{k} \cdot \mathbf{p}$ interactions between the conduction band $\Gamma_{6}$ and the valence bands $\Gamma_{8}$ and $\Gamma_{7}$ are fully, or exactly considered, i.e., not as a perturbation, ${ }^{9}$ and are free from any ambiguity connected to choices of operator ordering or spurious solutions.

\section{A. Perturbation expansion}

Consider now a heterojunction between semiconductors 1 $(z \leqslant 0)$ and $2(z \geqslant 0)$. Near the interface at $z=0$, the electrostatic potential energy is approximately given by $U(z)=e \mathcal{E} z$, with the uniform electric field given by $\mathcal{E}=e n_{s} / \varepsilon_{s c}$, where $n_{s}$ is electronic density of the $2 \mathrm{DEG}$ and $\varepsilon_{s c}$ is the dielectric constant. This is obtained by assuming that the system is infinite along the plane of the interface and by neglecting differences in $\varepsilon_{s c}$. The corresponding confining potential in the case of a CdTe/InSb heterojunction (in which the Rashba effect is particularly strong), with $n_{s}=2.0 \times 10^{11} \mathrm{~cm}^{-2}$ is shown in Fig. 1.

Despite its one-band appearance, Eq. (1) corresponds to the multiband effective mass equation, and must in general be solved by numerical integration, due to the energy and $z$ dependences on $m$ and $\beta$. However, for the lower lying electronic energy states in such heterojunctions, an amenable

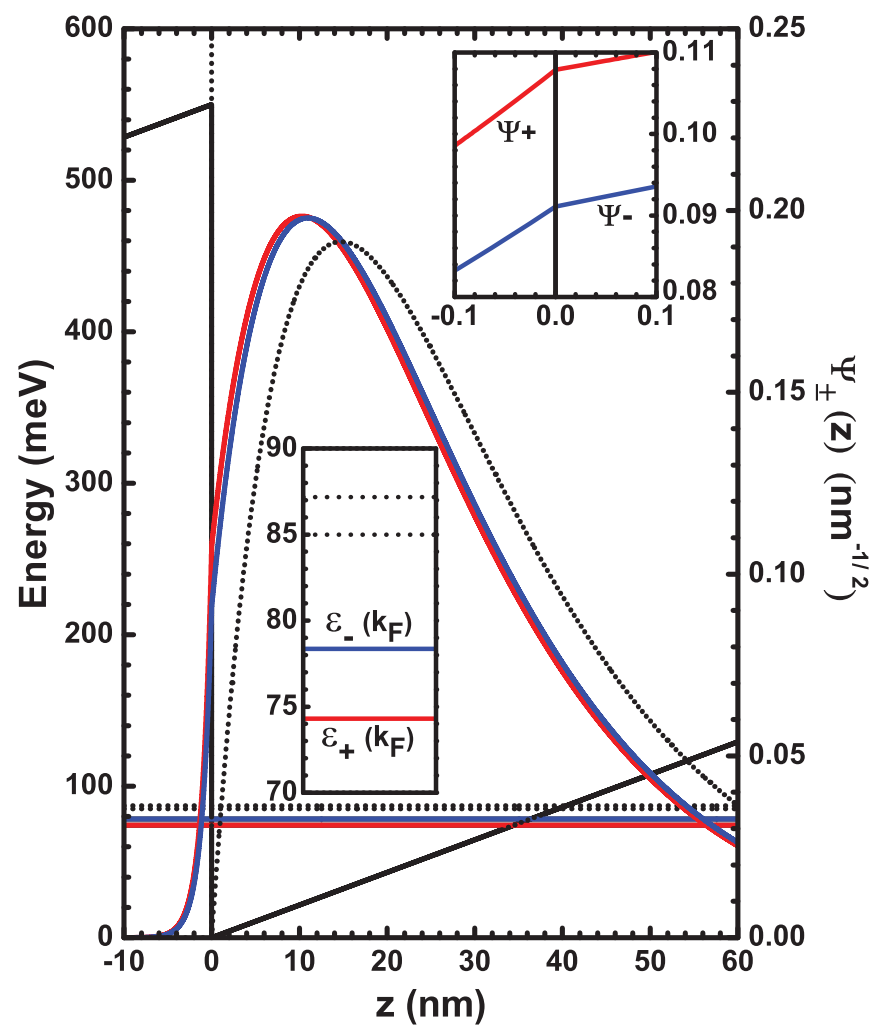

FIG. 1. (Color online) Confining potential, spin-dependent envelope functions, and spin-split energies at $k_{F}$ for a 2DEG in a $\mathrm{CdTe} / \mathrm{InSb}$ heterojunction, with $n_{s}=2.0 \times 10^{11} \mathrm{~cm}^{-2}$. The upper inset expands the interface region to better show the spin dependency of the envelope function. The lower inset shows the value of the Rashba splitting at the Fermi Level. The dotted lines correspond to the perfect insulating barrier approximation, i.e., insulator/InSb. 
form of the effective Hamiltonian can be obtained by choosing an appropriate small parameter $\delta$ to expand these quantities in a power series, i.e.,

$$
\frac{1}{m}=\sum_{n=0}^{\infty} \mathcal{A}_{n} \delta^{n} \text { and } \beta=\sum_{n=0}^{\infty} \mathcal{B}_{n} \delta^{n} .
$$

In material 2 , i.e., in the region $z \geqslant 0$, one can follow Ref. 6 and use $\delta=\left[\varepsilon_{ \pm}-U(z)\right] /\left(E_{g}+\Delta\right)$. In the present $\mathrm{CdTe} / \mathrm{InSb}$ heterojunction, the expectation value of $\delta$ in the first subband is estimated to be $\sim 0.025$ and terms of the order of $\delta^{2}$ will be neglected. By taking only the leading order terms, we obtain $m^{*}=\left(\hbar^{2} / P^{2}\right) E_{g}\left(E_{g}+\Delta\right) /\left(3 E_{g}+2 \Delta\right)$ and $\beta^{*}=\left(\hbar^{2} / 2 m^{*}\right) \Delta /\left(3 E_{g}+2 \Delta\right)$, in zeroth order, whereas the Rashba coupling parameter $\left(\alpha^{*}\right)$ appears only in first order, and is given by

$$
\alpha^{*}=\frac{\hbar^{2}}{2 m^{*}} \frac{\Delta}{E_{g}} \frac{2 E_{g}+\Delta}{\left(E_{g}+\Delta\right)\left(3 E_{g}+2 \Delta\right)} e \mathcal{E},
$$

as first obtained in Ref. 6.

In the barrier region instead, i.e., for $z \leqslant 0$, and since the energies of interest lay inside the gap, it is convenient to follow Ref. 10 and introduce renormalized barrier parameters $\bar{m}, \bar{\beta}$, and $\bar{\alpha}$, which include nonparabolicity corrections to the corresponding band-edge parameters, being though independent of the energy. The same procedure used in Ref. 10 with a $4 \times 4 \mathbf{k} \cdot \mathbf{p}$ model (without spin-orbit interaction) is followed here, now with the $8 \times 8$ Kane model and we obtain:

$$
\begin{aligned}
& \bar{m}=m^{*} \frac{\left(1-v_{0} / E_{g}\right)\left[1-v_{0} /\left(E_{g}+\Delta\right)\right]}{1-v_{0} /\left(E_{g}+2 \Delta / 3\right)}, \\
& \bar{\beta}=\beta^{*} \frac{1}{\left(1-v_{0} / E_{g}\right)\left[1-v_{0} /\left(E_{g}+\Delta\right)\right]},
\end{aligned}
$$

and

$$
\bar{\alpha}=\alpha^{*} \frac{1-v_{0} /\left(E_{g}+\Delta / 2\right)}{\left(1-v_{0} / E_{g}\right)^{2}\left[1-v_{0} /\left(E_{g}+\Delta\right)\right]^{2}} .
$$

Note that, as expected, they reduce exactly to their corresponding band-edge values, $m^{*}, \beta^{*}$, and $\alpha^{*}$, as the barrier height $v_{0}$ goes to zero.

Therefore, to leading order in the expansions, the effective Hamiltonian (1) is

$$
H_{\mathrm{eff} \pm}=\left\{\begin{array}{ll}
\frac{\hbar^{2}}{2 \bar{m}}\left(-\frac{d^{2}}{d z^{2}}+k_{\|}^{2}\right)+v_{0}+e \mathcal{E} z \mp \bar{\alpha} k_{\|}, & z \leqslant 0 \\
\frac{\hbar^{2}}{2 m^{*}}\left(-\frac{d^{2}}{d z^{2}}+k_{\|}^{2}\right)-a \frac{\left[\frac{\hbar^{2}}{2 m^{*}}\left(-\frac{d^{2}}{d z^{2}}+k_{\|}^{2}\right)\right]^{2}}{E_{g}+\Delta}+e \mathcal{E} z \mp \alpha^{*} k_{\|}, & z \geqslant 0,
\end{array}\right\},
$$

where the nonparabolicity constant

$$
a=\frac{2\left(E_{g}+\Delta\right)^{2}+E_{g}^{2}}{E_{g}\left(3 E_{g}+2 \Delta\right)} .
$$

In this form, for the calculation of the first subbands, $H_{\mathrm{eff} \pm}$ is seen to keep all the physics and most of the accuracy of the Kane model, but in a simple single-band form (not dependent on $\varepsilon_{ \pm}$) and therefore amenable to a variational solution. The boundary condition (4) is now given by

$$
-\frac{\hbar^{2}}{2 \bar{m}} \frac{d}{d z} \Psi_{1 \pm} \mp \bar{\beta} k_{\|} \Psi_{1 \pm}=-\frac{\hbar^{2}}{2 m^{*}} \frac{d}{d z} \Psi_{2 \pm} \mp \beta^{*} k_{\|} \Psi_{2 \pm} .
$$

Note that slightly different perturbation expansions are used in each side of the interface, and that our results are correct only to the leading order in each side. Accordingly, the boundary conditions in Eq. (4) are, here also, satisfied only to this approximation. The model band parameters are listed in Table I. For $z \geqslant 0$, they are those of the well material: $m^{*}=m^{*(2)}, E_{g}=E_{g}^{(2)}$, and $\Delta=\Delta^{(2)}$. For $z \leqslant 0$, corresponding to the barrier, $E_{g}=E_{g}^{(1)}$ and $\Delta=\Delta^{(1)}$, while $m^{*}=$ $m^{*(1)}=\left(\hbar^{2} / P^{2}\right) E_{g}^{(1)}\left(E_{g}^{(1)}+\Delta^{(1)}\right) /\left(3 E_{g}^{(1)}+2 \Delta^{(1)}\right)$, used in the calculation of $\bar{m}, \bar{\alpha}$, and $\bar{\beta}$, is obtained with the same value of $P$ determined by $m^{*(2)}$. Finally, the band offset or barrier height is $v_{0}=E_{c}^{(1)}-E_{c}^{(2)}$ and $E_{c}^{(2)}=0$ we set as the energy origin, in the figures.

\section{SPIN-DEPENDENT VARIATIONAL APPROACH}

Due to the above boundary conditions, as opposed to the infinite barrier approximation, ${ }^{6}$ the trial functions of a variational solution must be spin dependent when there is barrier penetration, and the eigenenergies will be given by

$$
\varepsilon_{ \pm}\left(k_{\|}\right)=\left\langle\Psi_{ \pm}\left|H_{\mathrm{eff} \pm}\right| \Psi_{ \pm}\right\rangle=\left\langle H_{\mathrm{eff} \pm}\right\rangle_{ \pm} .
$$

The effective Hamiltonian (10) can be written as a sum of four different terms, $H_{\mathrm{eff} \pm}=\sum_{i=1}^{4} H_{\mathrm{eff} \pm}^{(i)}$, so to have

$$
\varepsilon_{ \pm}\left(k_{\|}\right)=T_{ \pm}+T_{n p \pm}+V_{ \pm}+V_{s o \pm}
$$

where

$$
\begin{gathered}
T_{ \pm}=\left\langle\frac{\hbar^{2}}{2}\left(\frac{1}{\bar{m}^{(1)}} \theta(-z)+\frac{1}{m^{*(2)}} \theta(z)\right)\left(-\frac{d^{2}}{d z^{2}}+k_{\|}^{2}\right)\right\rangle_{ \pm} \\
T_{n p \pm}=\left\langle-a \frac{\left[\frac{\hbar^{2}}{2 m^{*(2)}}\left(-\frac{d^{2}}{d z^{2}}+k_{\|}^{2}\right)\right]^{2}}{E_{g}^{(2)}+\Delta^{(2)}} \theta(z)\right\rangle_{ \pm} \\
V_{ \pm}=\left\langle e \mathcal{E} z+v_{0} \theta(-z)\right\rangle_{ \pm}
\end{gathered}
$$

and

$$
V_{s o \pm}=\left\langle\mp\left(\bar{\alpha} \theta(-z)+\alpha^{*} \theta(z)\right) k_{\|}\right\rangle_{ \pm} .
$$

These contributions can be further separated into barrier and well components, i.e., $T_{ \pm}=\bar{T}_{ \pm}+T_{ \pm}^{*}$, and similarly for $V$ and $V_{\text {so }}$. 
TABLE I. Heterojunction bulk band parameters (Refs. 13-15) used in the present calculations.

\begin{tabular}{|c|c|c|c|c|c|c|c|}
\hline & $E_{g}^{(1)}(\mathrm{eV})$ & $\Delta^{(1)}(\mathrm{eV})$ & $v_{0}(\mathrm{meV})$ & $E_{g}^{(2)}(\mathrm{eV})$ & $\Delta^{(2)}(\mathrm{eV})$ & $m^{*(2)}\left(m_{e}\right)$ & $\varepsilon_{s c}$ \\
\hline $\mathrm{CdTe} / \mathrm{InSb}$ & 1.590 & 0.800 & 550 & 0.240 & 0.810 & 0.015 & 16.8 \\
\hline Insulator/InAs & $\ldots$ & $\ldots$ & $\infty$ & 0.418 & 0.380 & 0.023 & 12.2 \\
\hline $\mathrm{InP} / \mathrm{In}_{0.53} \mathrm{Ga}_{0.47} \mathrm{As}$ & 1.423 & 0.107 & 250 & 0.813 & 0.326 & 0.041 & 13.1 \\
\hline $\mathrm{In}_{0.52} \mathrm{Al}_{0.48} \mathrm{As} / \mathrm{In}_{0.53} \mathrm{Ga}_{0.47} \mathrm{As}$ & 1.513 & 0.309 & 500 & 0.813 & 0.326 & 0.041 & 13.1 \\
\hline $\mathrm{Al}_{0.3} \mathrm{Ga}_{0.7} \mathrm{As} / \mathrm{GaAs}$ & 1.893 & 0.334 & 269 & 1.519 & 0.340 & 0.067 & 12.9 \\
\hline
\end{tabular}

For a given electron density $n_{s}$, the trial function $\Psi_{ \pm}$or its variational parameter is determined by minimizing the total energy (sum over all the electrons). Assuming all the electrons in the first subband, such minimization of the total energy in the Hartree approximation for the electron-electron (e-e) interaction corresponds to minimizing $\tilde{\varepsilon}_{ \pm}$, equal to the above single particle energy $\varepsilon_{ \pm}$except for the substitution in $V_{ \pm}$of the electric field $\mathcal{E}$ by $\mathcal{E} / 2$ due to the double count in the e-e interaction. ${ }^{11}$

From the obtained energy splitting $\left(\delta \varepsilon=\left\langle H_{\mathrm{eff}+}\right\rangle_{+}-\right.$ $\left.\left\langle H_{\text {eff- }}\right\rangle_{-}\right)$of the states at the Fermi level, we define an effective Rashba coupling parameter by setting

$$
|\delta \varepsilon|=2 \alpha_{\text {eff }} k_{F} \text {. }
$$

In the present scheme, the Rashba splitting is then given as a sum of different contributions, i.e., $\delta \varepsilon=\sum_{i} \delta \varepsilon_{i}$, with

$$
\delta \varepsilon_{i}=\left\langle H_{\mathrm{eff}+}^{(i)}\right\rangle_{+}-\left\langle H_{\mathrm{eff}-}^{(i)}\right\rangle_{-},
$$

where $i$ labels the four contributions of Eqs. (15)-(18).

\section{A. Spin-dependent trial functions}

Calculations based on the celebrated Fang-Howard variational function have been intensively applied to the study of semiconductor heterojunctions. ${ }^{1-13}$ We here introduce modified spin-dependent Fang-Howard trial functions to describe Rashba split 2DEGs in III-V semiconductor heterojunctions. They satisfy the above boundary conditions, allow for a simple lowest subband solution, including the Rashba spin-orbit coupling, band nonparabolicity and barrier penetration effects, and read:

$$
\Psi_{ \pm}(z)= \begin{cases}\Psi_{1 \pm}=A_{ \pm} e^{k_{b} z / 2}, & z \leqslant 0 \\ \Psi_{2 \pm}=B_{ \pm}\left(z+c_{ \pm}\right) e^{-b z / 2}, & z \geqslant 0\end{cases}
$$

where $k_{b}=2 \sqrt{2 \bar{m}^{(1)} v_{0} / \hbar^{2}}$ and $b$ is the variational parameter determined by minimizing the total energy.

As can be straightforwardly verified, the boundary condition (12) implies that

$$
A_{ \pm}=B_{ \pm} c_{ \pm}
$$

and

$$
c_{ \pm}=\frac{2}{b+\frac{m^{*(2)}}{\bar{m}^{(1)}} k_{b} \pm 4 \frac{m^{*(2)}}{\hbar^{2}}\left(\bar{\beta}^{(1)}-\beta^{*(2)}\right) k_{\|}},
$$

which explicitly show the analytical coupling between the spin and the dynamic variable $k_{\|}$of the $2 \mathrm{D}$ electrons. From the normalization condition, we obtain

$$
B_{ \pm}=\sqrt{\frac{b^{3} / 2}{1+b c_{ \pm}+b^{2} c_{ \pm}^{2}\left(1+b / k_{b}\right) / 2}} .
$$

The spin-dependent density of probability in the regions 1 and 2 are, respectively, $\left\langle\Psi_{1 \pm} \mid \Psi_{1 \pm}\right\rangle=A_{ \pm}^{2} / k_{b}$ and $\left\langle\Psi_{2 \pm} \mid \Psi_{2 \pm}\right\rangle=B_{ \pm}^{2}\left(2 / b^{3}+2 c_{ \pm} / b^{2}+c_{ \pm}^{2} / b\right)$. The Eqs. (22), (23), and (24) completely determine $A_{ \pm}, B_{ \pm}$, and $c_{ \pm}$in terms of $b$ and $k_{b}$.

For the expectation value of the kinetic energy in the two sides of the interface, we obtain:

$$
\bar{T}_{ \pm}=\frac{\hbar^{2}}{2 \bar{m}^{(1)}}\left(-\frac{A_{ \pm}^{2} k_{b}}{4}+\left\langle\Psi_{1 \pm} \mid \Psi_{1 \pm}\right\rangle k_{\|}^{2}\right)
$$

and

$$
T_{ \pm}^{*}=\frac{\hbar^{2}}{2 m^{*(2)}}\left[B_{ \pm}^{2}\left(\frac{1}{2 b}+\frac{c_{ \pm}}{2}-\frac{1}{4} b c_{ \pm}^{2}\right)+\left\langle\Psi_{2 \pm} \mid \Psi_{2 \pm}\right\rangle k_{\|}^{2}\right] .
$$

The nonparabolicity correction in the well region becomes

$$
\begin{aligned}
T_{n p \pm}^{*}= & -\frac{a}{E_{g}^{(2)}+\Delta^{(2)}} \frac{\hbar^{4}}{4\left(m^{*(2)}\right)^{2}}\left[\frac{B_{ \pm}^{2} b}{8}\left(\frac{1}{2} c_{ \pm}^{2} b^{2}-3 c_{ \pm} b-3\right)\right. \\
& \left.+B_{ \pm}^{2}\left(\frac{1}{b}+c_{ \pm}-\frac{b c_{ \pm}^{2}}{2}\right) k_{\|}^{2}+\left\langle\Psi_{2 \pm} \mid \Psi_{2 \pm}\right\rangle k_{\|}^{4}\right] .
\end{aligned}
$$

For the expectation values of the potential energy we obtain:

$$
\bar{V}_{ \pm}=\frac{A_{ \pm}^{2}}{k_{b}}\left(v_{0}-\frac{e \mathcal{E}}{k_{b}}\right)
$$

and

$$
V_{ \pm}^{*}=e \mathcal{E} B_{ \pm}^{2}\left(\frac{6}{b^{4}}+4 \frac{c_{ \pm}}{b^{3}}+\frac{c_{ \pm}^{2}}{b^{2}}\right) .
$$

Finally, the components of the direct spin-orbit coupling energy read are

$$
\begin{gathered}
\bar{V}_{s o \pm}=\mp \bar{\alpha}\left\langle\Psi_{1 \pm} \mid \Psi_{1 \pm}\right\rangle k_{\|}, \\
V_{s o \pm}^{*}=\mp \alpha^{*}\left\langle\Psi_{2 \pm} \mid \Psi_{2 \pm}\right\rangle k_{\|} .
\end{gathered}
$$

\section{RESULTS FOR DIFFERENT III-V HETEROJUNCTIONS}

The upper inset in Fig. 1 shows the small envelope function amplitude and derivative discontinuity at the interface, obtained for electrons with opposite spins. However, 


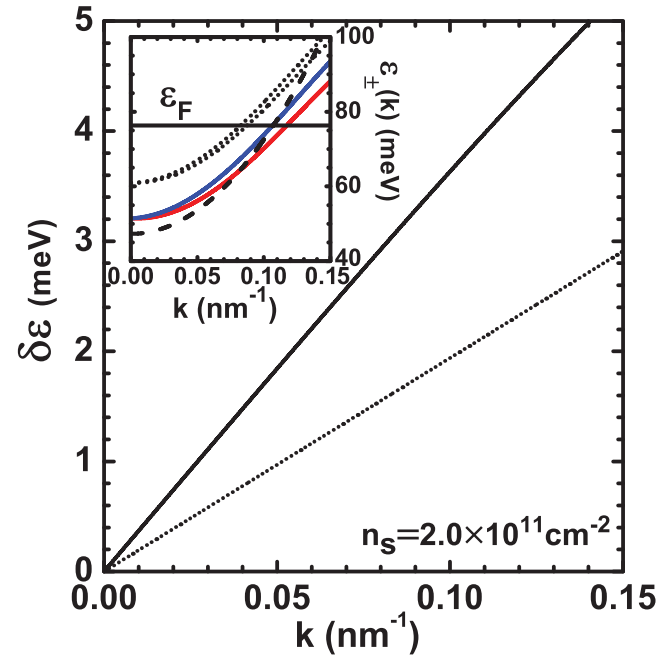

FIG. 2. (Color online) Rashba splitting as a function of the parallel wave vector. Inset shows the spin-split conduction subband dispersion relations and parabolic approximation (dashed line) for a $\mathrm{CdTe} / \mathrm{InSb}$ heterojunction. The dotted lines show the limiting case of insulator/InSb.

as shown in the lower inset, these small differences have a considerable effect in the corresponding energy levels and in the Rashba splitting (the dotted lines show the results for an infinite barrier). For the heterojunction in Fig. 1, we obtain $b=0.14 \mathrm{~nm}^{-1}$ independent of $k_{\|}$and spin. Figure 2 shows the corresponding Rashba splitting as a function of the parallel wavevector, and the inset shows the subband dispersion relations, where the dashed line represents the parabolic spin-independent limit. We see that for the present $\mathrm{CdTe} / \mathrm{InSb}$ heterojunction, barrier penetration is predicted to be responsible for an increase close to $85 \%$ in the Rashba splitting relative to the infinite barrier approximation.

Such strong barrier penetration effect can be analyzed by decomposing the Rashba splitting into its different contributions, as plotted in Fig. 3 for the heterojunction of Fig. 1. It is interesting to note that all the different terms of the effective Hamiltonian do contribute to the total spin splitting. In particular, we also see that the contributions from $\bar{T}$ and $T^{*}$ are the main ones responsible for the above-mentioned increase in the Rashba splitting, as the contributions of $\bar{V}$ and $V^{*}$ nearly cancel each other and those of $\bar{V}_{s o}$ and $T_{n p}^{*}$ are negligible. For CdTe/InSb heterojunctions with $n_{s}=2.0 \times 10^{11} \mathrm{~cm}^{-2}$, for example, we obtain a Rashba splitting at the Fermi level $\delta \varepsilon=4.1 \mathrm{meV}$, which, in $\mathrm{meV}$, is the sum of $\delta T^{*}=0.7$, $\delta \bar{T}=1.0, \delta T_{n p}^{*}=-0.1, \delta V^{*}=1.3, \delta \bar{V}=-1.0, \delta V_{s o}^{*}=2.2$, and $\delta \bar{V}_{s o} \sim 0$. For an infinite barrier, only $\delta V_{s o}^{*}$ remains; all the other contributions go to zero. In this limit, the envelope function becomes independent of spin, and no Rashba splitting contribution is obtained from the kinetic or potential energy terms, so that we get $\alpha_{\text {eff }}=\alpha^{*}$. Therefore, for $v_{0} \rightarrow \infty$, we have: $k_{b} \rightarrow \infty, c_{ \pm} \rightarrow 0,\left\langle v_{0} \theta(-z)\right\rangle_{ \pm} \rightarrow 0, B_{ \pm}^{2} \rightarrow b^{3} / 2$, and $\langle z\rangle_{ \pm} \rightarrow 3 / b$, which substituted in the above expressions for $\varepsilon\left(k_{\|}\right)$lead to the known infinite barrier results of Ref. 6. It is also interesting to note in Fig. 3 how the different contributions vary with the electron density. We can see, for instance, that the usual or direct Rashba spin-orbit term in the effective

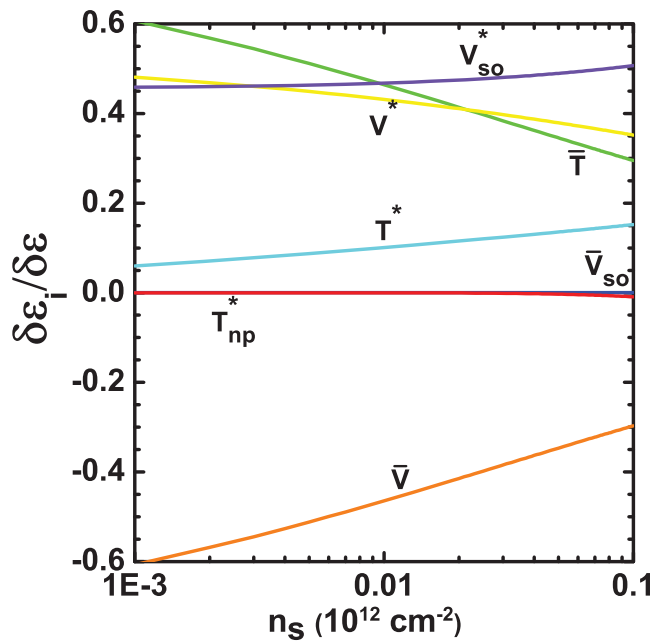

FIG. 3. (Color online) Partial contributions to the Rashba splitting as a function of the 2DEG carrier concentration in a CdTe/InSb heterojunction.

Hamiltonian (i.e., $V_{s o}^{*}$ ) dominates the Rashba effect only in $\mathrm{CdTe} / \mathrm{InSb}$ inversion layers with more than $10^{10} \mathrm{e} / \mathrm{cm}^{2}$. At lower densities, it is the barrier penetration kinetic energy term that dominates the Rashba effect in this system. This is a direct result of the spin-dependent boundary conditions [Eq. (12)].

\section{A. Spin-split subband population}

The Rashba coupling parameter at the Fermi level $\left(\varepsilon_{F}\right)$ can be estimated as $\alpha_{\text {eff }}=\left|\varepsilon_{+}\left(k_{F}\right)-\varepsilon_{-}\left(k_{F}\right)\right| / 2 k_{F}$, with $k_{F}=$ $\left(k_{+}+k_{-}\right) / 2$ and $k_{ \pm}$determined from $\varepsilon_{F}=\varepsilon_{+}\left(k_{+}\right)=\varepsilon_{-}\left(k_{-}\right)$. However, it is easier and usual to experimentally study the Rashba effect by measuring the difference in the split subband populations, directly from the beating pattern of the $\mathrm{SdH}$ oscillations. The populations $n_{-}$and $n_{+}$in each Rashba split subband, with $n_{+}+n_{-}=n_{s}$, are simply given by

$$
n_{ \pm}=\frac{1}{(2 \pi)^{2}} \int d \mathbf{k} \theta\left[\varepsilon_{F}-\varepsilon_{ \pm}(k)\right]=\frac{k_{ \pm}^{2}}{4 \pi} .
$$

The results for the population difference in the two split subbands (some times referred as the density spin polarization) for the different III-V heterojunctions are shown in Fig. 4(a) as a function of $n_{s}$. The parameters used are listed in Table I. Figure 4(b) shows the corresponding effective Rashba coupling parameter $\alpha_{\text {eff }}$. By comparing the InGaAs results which correspond to different barriers, we see that the larger penetration in the smaller InP barrier for the electrons leads to a considerable increase in the Rashba splitting, as compared to the InAlAs barrier. Figure 4 also clearly shows the well-known trend to larger splittings in heterojunctions formed by materials with smaller energy gaps. These results agree well with those obtained by numerical integration of the multiband effective Schroedinger equation and with available experimental data. ${ }^{5,16-19}$ As expected, such agreement gets worse as $n_{s}$ increases and the present scheme starts to overestimate the splitting. Anyway, before a detailed comparison with experiment is carried out, a Dresselhaus or bulk spin-orbit term must be added, specially for heterojunctions of large band gap materials, and for noncommon atom interfaces, the interface 

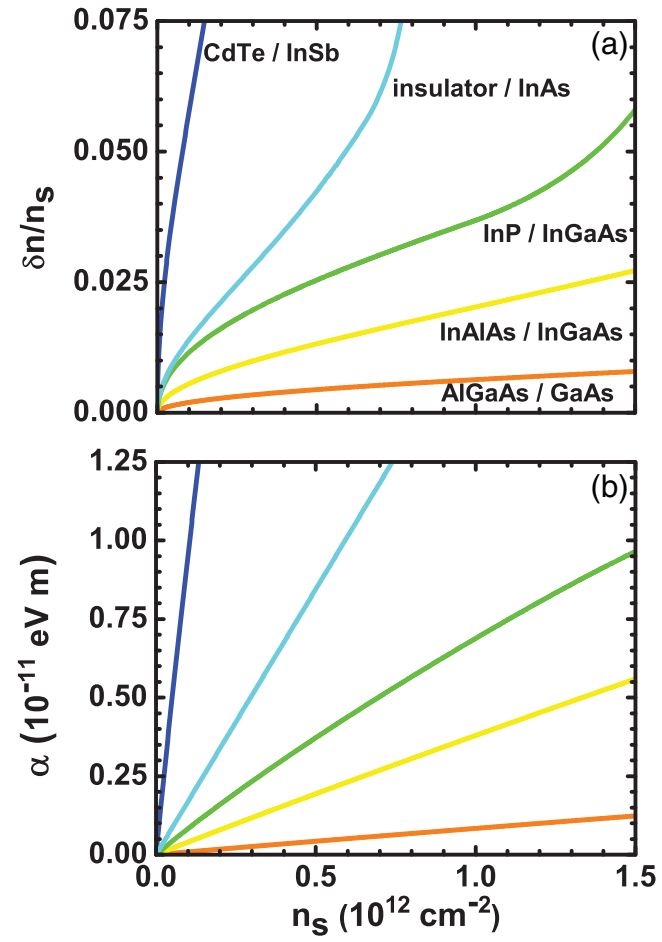

FIG. 4. (Color online) Spin-split subband population difference (a) and the resulting effective Rashba coupling parameter (b), as a function of the carrier density $n_{s}$ and for different III-V heterojunctions.

inversion asymmetry contribution ${ }^{20}$ must also be taken into account.

\section{CONCLUSIONS}

We have performed a variational analysis of the Rashba spin-orbit splitting in III-V semiconductor inversion layers. The Rashba coupling parameter and the corresponding subband population difference, as experimentally determined from the beating pattern of the $\mathrm{SdH}$ oscillations, are calculated for inversion layers at $\mathrm{CdTe} / \mathrm{InSb}$, insulator/InAs, InP/InGaAs, InAlAs/InGaAs, and $\mathrm{AlGaAs} / \mathrm{GaAs}$ heterojunctions. The main properties of the Rashba effect are shown to be well reproduced by the theory. It is also shown that the separate contributions to the Rashba effect in III-V heterojunctions can be studied with accurate analytical expressions. In particular, the explicit dependence on the different heterostructure and bulk band parameters can be very useful in device development and optimization. The present results also help to clarify the controversial role of the interface and barrier penetration. It is clearly seen, for example, the increasing importance of the spin-dependent boundary conditions as the 2DEG electron density is reduced. In CdTe/InSb inversion layers, for example, the usual (or direct) Rashba spin-orbit term in the effective Hamiltonian is seen to dominate the Rashba effect only for $n_{s}>10^{10} \mathrm{~cm}^{-2}$. Finally, it is worth mentioning that the theory details presented here, as well as the intermediate analytical results for the energy integrals [Eqs. (25)-(31)] and the instructions for correct use of the parameters, allow the present theory to be easily applied in the study of the Rashba effect in any other III-V semiconductor heterojunction.

\section{ACKNOWLEDGMENTS}

The authors wish to thank the brazilian agencies CNPq and FAPESB for financial support, and H. Closs for the revision. *erasmo@las.inpe.br

${ }^{1}$ D. Awschalom and N. Samarth, Physics 2, 50 (2009).

${ }^{2}$ I. Zutic, J. Fabian, and S. D. Sarma, Rev. Mod. Phys. 76, 323 (2004).

${ }^{3}$ U. Ekenberg and D. M. Gvozdic, Phys. Rev. B 78, 205317 (2008).

${ }^{4} \mathrm{R}$. Winkler, in Spin-Orbit Coupling Effects in Two-Dimensional Electron and Hole Systems, Springer Tracts in Modern Physics Vol. 191 (Springer, Berlin, 2003).

${ }^{5}$ M. A. Toloza Sandoval, A. Ferreira da Silva, E. A. de Andrada e Silva, and G. C. La Rocca, Phys. Rev. B 79, 241305(R) (2009).

${ }^{6}$ E. A. de Andrada e Silva, G. C. La Rocca, and F. Bassani, Phys. Rev. B 50, 8523 (1994).

${ }^{7}$ G. Marques and L. Sham, Surf. Sci. 113, 131 (1982).

${ }^{8}$ L. G. Gerchikov and A. V. Subashiev, Sov. Phys. Semicond. 26, 73 (1992).

${ }^{9}$ D. G. Rothe, R. W. Reinthaler, C.-X. Liu, L. W. Molenkamp, S.-C. Zhang, and E. M. Hankiewicz, New J. Phys. 12, 065012 (2010).

${ }^{10}$ G. Brozak, E. A. de Andrada e Silva, L. J. Sham, F. DeRosa, P. Miceli, S. A. Scharwz, J. P. Harbison, L. T. Flores, and S. J. Allen, Phys. Rev. Lett. 64, 471 (1990).
${ }^{11}$ G. Bastard, Wave Mechanics Applied to Semiconductor Heterostructures (Les editions de physique, Les Ulis, 1990).

${ }^{12}$ F. Fang and W. Howard, Phys. Rev. Lett. 16, 18 (1966).

${ }^{13}$ J. Davies, The Physics of Low-Dimensional Semiconductors: An Introduction (Cambridge University Press, Cambridge, 1998).

${ }^{14}$ J.-M. Jancu, R. Scholz, E. A. de Andrada e Silva, and G. C. La Rocca, Phys. Rev. B 72, 193201 (2005).

${ }^{15}$ Landolt-Bornstein New Series, edited by O. Madelung (Springer, Berlin, 1987), Vol. III/22a.

${ }^{16}$ E. A. de Andrada e Silva, G. C. La Rocca, and F. Bassani, Phys. Rev. B 55, 16293 (1997).

${ }^{17}$ T. Matsuyama, R. Kursten, C. Meissßner, and U. Merkt, Phys. Rev. B 61, 15588 (2000).

${ }^{18}$ C. Schierholz, T. Matsuyama, U. Merkt, and G. Meier, Phys. Rev. B 70, 233311 (2004).

${ }^{19}$ C. L. Yang, H. T. He, L. Ding, L. J. Cui, Y. P. Zeng, J. N. Wang, and W. K. Ge, Phys. Rev. Lett. 96, 186605 (2006).

${ }^{20}$ L. Vervoort, R. Ferreira, and P. Voisin, Phys. Rev. B 56, R12744 (1997). 